\title{
MATHEMATICAL MODELING OF STALK BIOMASS SHREDDING PROCESS
}

\author{
Imants Nulle, Aivars Kakitis, Mareks Smits, Olafs Vronskis \\ Latvia University of Life Sciences and Technologies, Latvia \\ imants.nulle@1lu.1v, aivars.kakitis@1lu.lv, mareks.smits@1lu.lv, olafs.vronskis@1lu.lv
}

\begin{abstract}
In Latvia, approximately $14.6 \%$ of unfarmed agricultural land can be used for herbaceous energy crop growing. Herbaceous energy crops would be the main basis for solid biofuel production in the agricultural ecosystem in future. There is a possibility to utilize for bioenergy production natural biomass of common reeds (Phragmites Australis), overgrowing the shorelines of Latvian more than 2000 lakes. Common reed stalk material ultimate tensile strength average value was stated $330 \pm 29 \mathrm{~N} \cdot \mathrm{mm}^{-2}$. This value testifies that common reeds are the strongest material among other energy crop stalk materials. The main conditioning operation before preparation of herbaceous biomass compositions for solid biofuel production is shredding. In the previous experiments it was stated that cutting energy depends on the cutting knife angles against the shear bar, layer thickness of the material and the shape of the knives. It is important to designate the significance of these parameters to biomass shredder design. In the article a mathematical model of the stalk biomass cutting process is described, which evaluates the energy consumption dependence on the angle between the knife and the shear bar, distance of the knife from the rotor centre and thickness of the cutting layer. The model includes the peculiarities of the shredder rotor design, which determines the regularity of the angle of the knife angle relative to the counter cutter, depending on the rotor rotation angle. The obtained results will allow to determine the most suitable positioning of the knives on the rotor, ensuring smooth torque in the cutting process.
\end{abstract}

Keywords: cutting energy, common reeds, modelling, biomass shredder.

\section{Introduction}

Researchers from the Latvia University of Life Sciences and Technologies, Faculty of Engineering, Institute of Mechanics have studied the grinding and cutting processes of stalk biomass materials. [1-6]. The effect of the knife bevel and blade angle, cutting layer thickness, cutting force and the energy required for chopping have been studied. Rich data are obtained from the common reed cutting process. Phragmites australis (common reed) is a typical wetland vegetation type, it is also considered as an aggressive vegetation invader in the regional ecosystem [7]. As the common reed resources in Latvia are large [8;9], they can be used to produce solid biofuels. This would prevent overgrowing of lakes and improve the sustainability of the ecosystem. The obtained data must be compiled in a mathematical model that would allow obtain the dynamic and power consumption parameters of the reed shredder. The knowledge gained from the experimental studies will allow creation of an effective cutting mechanism.

Manufacturers include automatic systems and sensors for adjusting the gap between the knives and the shear bar. If the knife to the shear bar clearance changes from 0.1 to $0.7 \mathrm{~mm}$, the cutting energy increases three times. Cutting energy is also heavily affected by the knife sharpness, if the knife becomes blunt, then the cutting energy can double. Therefore, automatic knife sharpening mechanisms are increasingly used in agricultural machinery. This also determines the cutting cylinder design (Fig. 1). There are two main options for arranging the knives in the cutting cylinder: V-shape and multi-knife systems [10].

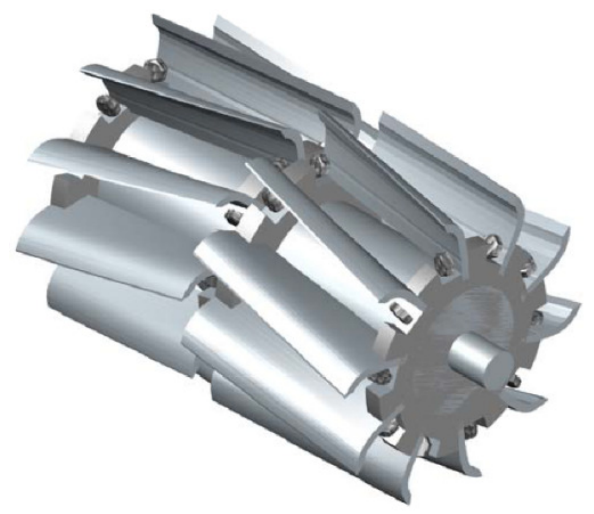

Fig. 1. Cutting cylinder 
If the knives are placed in the $\mathrm{V}$-shape, they are not removed from the cylinder by sharpening, thereby obtaining an elliptical knife shape. The mathematical shredder rotor model will be developed by taking into account this elliptical shape of the knife, as well as the angle of the knife bevel and blade angle. Also, the properties of the material to be chopped will be considered in the model and the energy required for common reed flattening and cutting, which depends on the thickness of the biomass layer and the knife geometric parameters.

\section{Materials and methods}

The position of the knife is important to determine the cutting parameters. It is determined by the intersection of the cutting cylinder and the knife plane; the shape of the knife is an ellipse (Fig. 2).

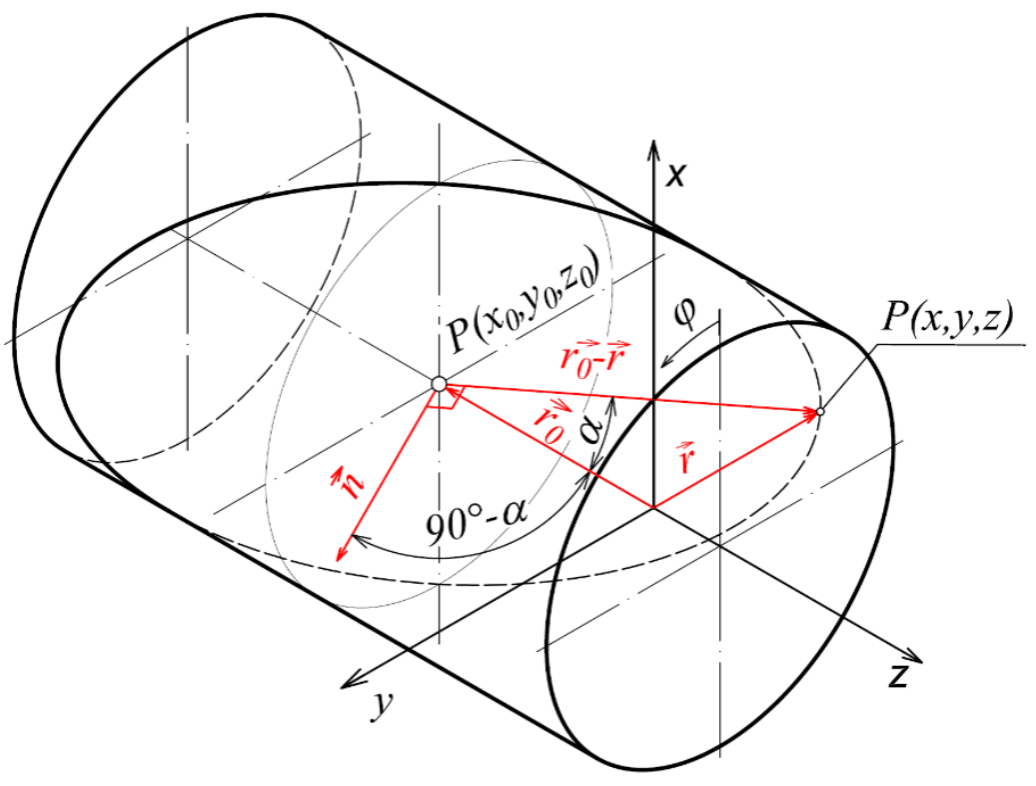

Fig. 2. Geometric model of cutting cylinder

Vector equation of the knife plane is:

$$
\left(\vec{r}-\vec{r}_{0}\right) \cdot \vec{n}=0,
$$

where $\vec{r}=\langle x, y, z\rangle$;

$$
\begin{aligned}
& \vec{r}_{0}=\langle x, y, z\rangle ; \\
& \vec{n}=a \cdot \vec{i}+b \cdot \vec{j}+c \cdot \vec{k} \text { or }\langle a, b, c\rangle .
\end{aligned}
$$

Scalar equation of the knife plane is:

$$
z=-\frac{b \cdot y}{c}+z_{0}=-y \cdot \operatorname{tg}\left(90^{\circ}-\alpha\right)+z_{0},
$$

Since the cutting cylinder is symmetric around the $\mathrm{z}$ axis, it may be easier to express the curve in cylindrical coordinates.

$$
x=r \cdot \cos \varphi ; \quad y=r \cdot \sin \varphi ; z=z .
$$

The curve of intersection may be parametrized as a function of $r$ and $\varphi$ :

$$
z=-r \cdot \sin \varphi \cdot \operatorname{tg}\left(90^{\circ}-\alpha\right)+z_{0}
$$

The model also provides obtaining an angle $\gamma$ of the knife with the shear bar (Fig. 3). The angle $\gamma$ will change faster, if the plane angle $\alpha$ is greater (Fig 2). In practice, cylinders with slight changes in the angle of the knife $\gamma$ in relation to the shear bar are used. In the model, the angle $\gamma$ is defined as the angle between two lines defined by 3 points (Fig. 3). Point A is a freely chosen intersection of the knife and the shear bar at a moment in time. Point B is the point of intersection of the knife and the 
shear bar after a certain time. However, point $\mathrm{C}$ is the shift of point $\mathrm{A}$ at the same time, without changing the coordinate $z$. The angle between the lines BC and BA is calculated as follows:

$$
\cos \gamma=\overrightarrow{B C} \cdot \overrightarrow{B A}=\left|\frac{\left(a_{1} \cdot \vec{i}+b_{1} \cdot \vec{j}+c_{1} \cdot \vec{k}\right) \cdot\left(a_{2} \cdot \vec{i}+b_{2} \cdot \vec{j}+c_{2} \cdot \vec{k}\right)}{\sqrt{a_{1}^{2}+b_{1}^{2}+c_{1}^{2}} \cdot \sqrt{a_{2}^{2}+b_{2}^{2}+c_{2}^{2}}}\right|
$$

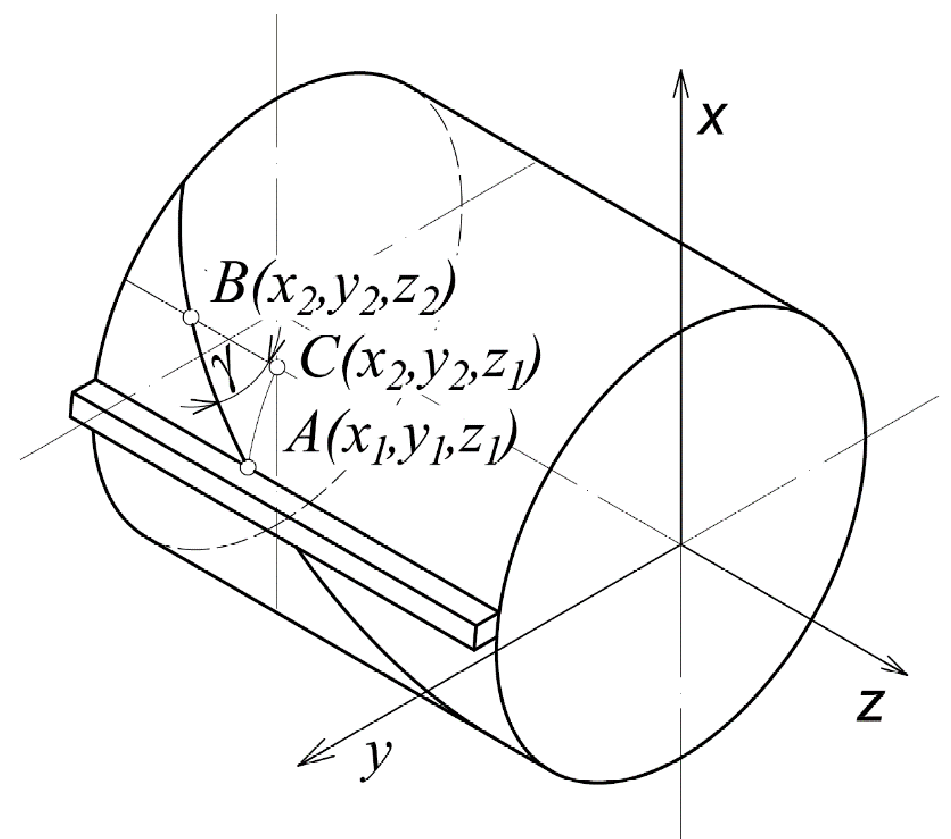

Fig. 3. Angle between knife and shear bar

In the cutting process, the stalk is initially flattened and only then cut. Therefore, the mathematical model also implements the energy required for flattening. The flattening force reaches a certain value and then cutting of the material begins.The width and thickness of the layer are affected by changes in the flattening force. To simplify the model, it can be assumed with a slight error that the flattening force of the common reed layer increase is linear. This is determined by the specific flattening force of the common reed stem determined experimentally [11]. The specific force value shows increase in the force per one meter of the knife displacement by flattening reed with a diameter of $0.01 \mathrm{~m}$. If the reeds are in several layers, then the flattening force will increase proportionally slower, it will be considered by the coefficient $k_{T H}$, but if the cutting layer is wider, the flattening force will increase proportionally faster, and it will be taken into account by the factor $k_{W}$.

Flattening force is calculated by the equation (6):

$$
F_{F L}(s)=\frac{F_{F L S} \cdot s \cdot k_{W}}{k_{T H}},
$$

where $F_{F L}(s)$ - flattening force, $\mathrm{N}$;

$F_{F L S}$ - specific flattening force, $\mathrm{N} \mathrm{mm}^{-1}$;

$s$ - displacement of the knife, m;

$k_{W}-$ coefficient of the width of the cutting layer;

$k_{T H}$ - coefficient of thickness of the cutting layer.

When modelling the cutting force, its variation depending on the angle of the blade $\left(k_{B L A}\right)$ [5], the angle of the bevel $\left(k_{B E A}\right)$ [12], the change in the cutting force depending on the thickness of the layer $\left(k_{T H F}\right)$ [12] is evaluated.In the formula it is estimated that about $3 / 4$ of the layer deformation is flattening and only $1 / 4$ is the cutting process $\left(k_{T H P}\right)[11 ; 12]$.

Cutting force is calculated by the equation (7): 


$$
\begin{gathered}
F_{C U}(s)=\frac{F_{C U S} \cdot s \cdot k_{W} \cdot k_{B E A} \cdot k_{B L A} \cdot k_{T H F}}{k_{T H}}-F_{C U S} \cdot k_{B E A} \cdot k_{B L A} \cdot k_{T H F} \cdot k_{W} \cdot k_{T H P}= \\
=F_{C U S} \cdot k_{B E A} \cdot k_{B L A} \cdot k_{T H F} \cdot k_{W} \cdot \frac{s-k_{T H} \cdot k_{T H P}}{k_{T H}}
\end{gathered}
$$

where $F_{C U}(s)$ - cutting force, $\mathrm{N}$;

$F_{\text {CUS }}$ - specificcutting force, $\mathrm{N} \mathrm{mm}^{-1}$

$s$ - displacement of the knife, m;

$k_{W}$-coefficientof the width of the cutting layer;

$k_{T H}$ - coefficientof thickness of the cutting layer;

$k_{B E A}$ - coefficient ofbevel angle of the knife;

$k_{B L A}$ - coefficientof the blade angle of the knife;

$k_{T H P}-$ coefficient of thickness proportion;

$k_{T H F}$ - coefficient of thickness influence on the cutting force.

Since the position of the knife is inclined, and when one part of the knife has finished cutting the layer, the other just starts, the cutting cylinder was divided into sections. The length of the section can be varied, but in this model it was $50 \mathrm{~mm}$. The power consumption and energy required for cutting was calculated for every section of the cutting cylinder. By integrating the function of force depending on the displacement of the knife, the energy needed for cutting was obtained. The model assumed that the flattening process took place $3 / 4$ of the knife displacement, and the cutting was $1 / 4$, ensuring a transition when the flattening force at that stage had reached the value of the cutting force.

$$
E_{C U}=\int_{s} F(s) d s,
$$

where $F(s)$ - flattening/cutting force, $\mathrm{N}$;

$E_{C U}$ - energy of flattening and cutting, $\mathrm{J}$;

$$
s=\omega \cdot t \cdot r,
$$

where $\omega$-rotation frequency, $\mathrm{s}^{-1}$;

$\gamma$ - radius of the cutting cylinder, $\mathrm{m}$.

$$
E_{C U}=\int_{t} F(t)(\omega \cdot t \cdot r)^{\prime} d t=\int_{t} F(t) \omega \cdot r \cdot d t,
$$

where $\varphi$-rotation angle of the cutting cylinder, rad.

\section{Results and discussion}

In this theoretical model, assuming that the cylinder is unlimited, we can achieve an ellipse at the intersection of the knife plane with the angle $\gamma$ of 0 to 90 degrees (Fig. 4). In practice, the cylinder has a limited length and the angle $\gamma$ of the model will also change within small limits, in aproximetly $2 \ldots 4$ degrees. An angle $\gamma$ (blade angle) of 10 degrees was used to obtain the data below.

Common reed layer thickness of $0.04 \mathrm{~m}$ was used to modeling flattening and shear force data. The knife length $0.5 \mathrm{~m}$ was divided into 10 sections, therefore the width of one section was $0.05 \mathrm{~m}$. The knife bevel angle was 90 degrees.

One section of $0.04 \times 0.05 \mathrm{~m}$ includes about 30 reeds with a diameter of $8 \mathrm{~mm}, 6$ reeds in the length of the section and 5 in height. The maximum force required to cut one reed is about $400 \mathrm{~N}$ [12]. It would be around $2400 \mathrm{~N}$ for 6 .

According to the authors' study [12], the force required for cutting reed increases up to 2 times if placed in several layers. Cutting is also affected by the blade and bevel angle of the knife. The multiplication factor can reach 2.6 times and it results to higher cutting force, as is the case with one reed cut by a knife with the bevel angle 20 degrees. For one knife section to move through the reed layer modelled force is shown in Fig. 5. The flattening force transforms to the cutting force until the 
reed layer is cut. The displacement of layer flattening is about $3 / 4$ from the overall displacement and $1 / 4$ is for cutting.

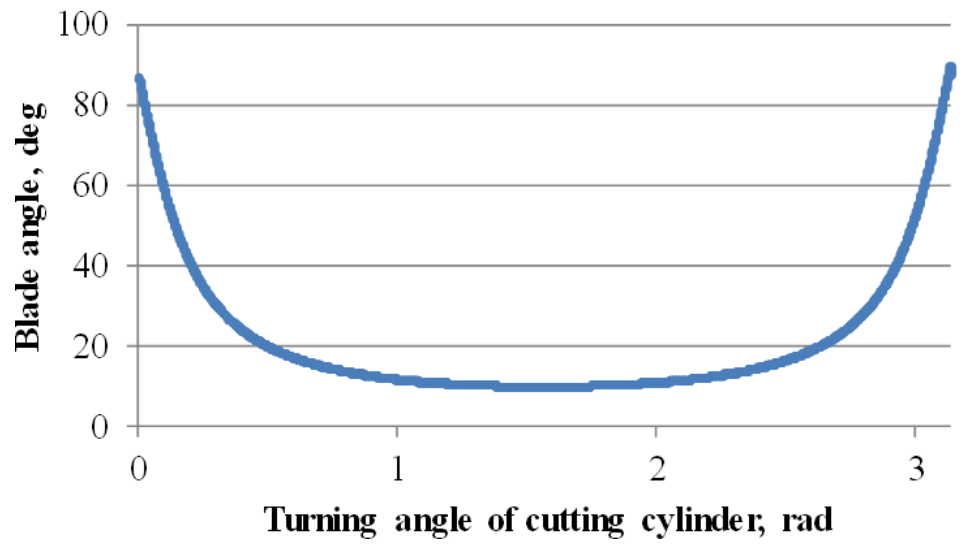

Fig. 4. Angle between knife and shear bar

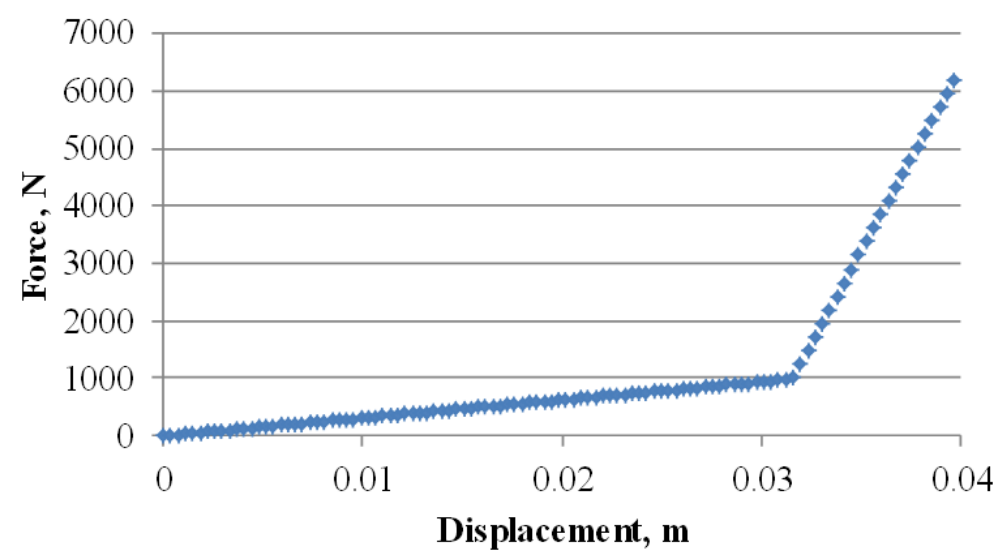

Fig. 5. Flattening/cutting force of one knife section

Cutting energy was calculated using the equation (10), and by cutting a $40 \mathrm{~mm}$ layer on one knife section $(50 \mathrm{~mm})$ it is $50 \mathrm{~J}$ (Fig. 6). Cutting one reed ( $8 \mathrm{~mm}$ diameter) in the biomass layer uses $1.5 \mathrm{~J}$ of energy, which is more than cutting separate reed stalks $0.2 \mathrm{~J}$ [12].

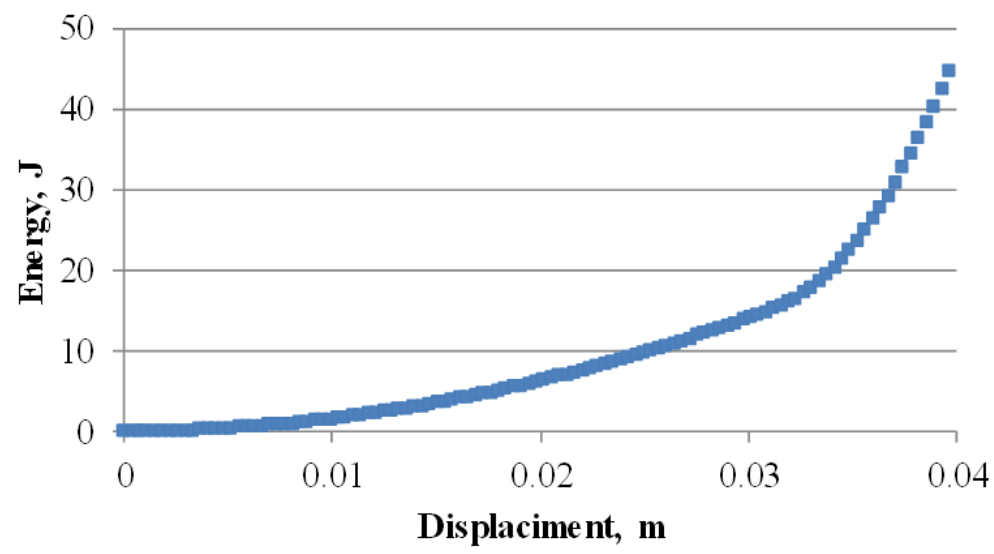

Fig. 6. Flattening/cutting energy of one knife section

The rotation frequency of the cutting cylinder was $1000 \mathrm{rpm}$. The power peak reached $300 \mathrm{~kW}$. The model does not include inertia of the cutting cylinder, it works as a flywheel and could reduce the power peaks to $200 \mathrm{~kW}$. The chart shows 10 sections of the knife sequentially. The knife is continuous, but the peculiarities of modelling divide the knife and also power into sections (Fig. 7). If more precise results are required, the knife must be divided into more sections. In practice, of course, the mass will not be completely smoothed out, so it is not possible to escape from the power peaks. 


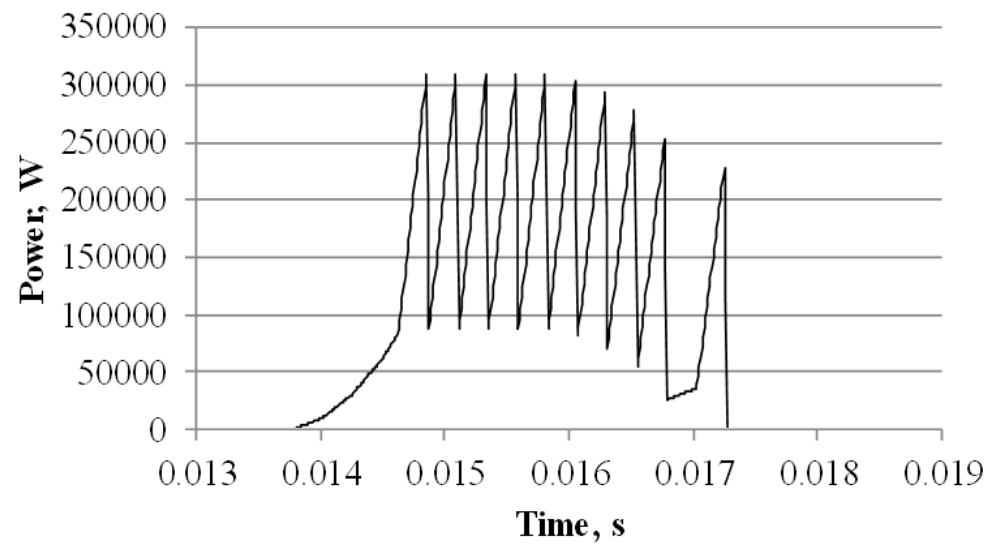

Fig. 7. Flattening/cutting power consumption for one cut (knife 10 sections)

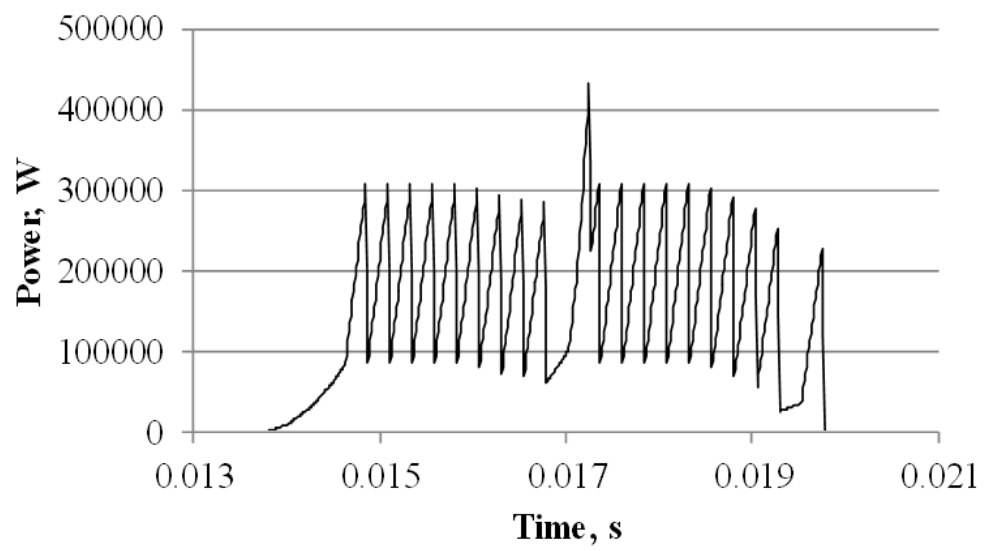

Fig. 8. Flattening/cutting power consumption for two following cuts

The options of the Matlab-Simulink model vary with the knife angles, rotation frequency, layer thickness, knife size, cylinder radius and width. It is possible to model the position of the knives so that the power peaks are minimal. Let one cut smoothly move to the next one (Fig. 8).

\section{Conclusions}

1. The blade angle of the cutting knife changes within small limits, in approximately $2 \ldots 4$ degrees.

2. The cutting force can increase 2.6 times depending on the angles of the knife, the thickness of the layer and other parameters.

3. The modelled power consumption of the rotor with the diameter $700 \mathrm{~mm}$ and rotating frequency $1000 \mathrm{rpm}$ by cutting a $40 \mathrm{~mm}$ thick and $500 \mathrm{~mm}$ wide reed layer is $300 \mathrm{~kW}$.

\section{References}

[1] Kronbergs A., Kronbergs E., Siraks E., Dalbins J. Cutting properties of arranged stalk biomass. Procceeding of International conference "Renewable energy and energy efficiency", May 28-30, 2012, Jelgava, Latvia, pp. 145-149.

[2] Kronbergs E., Smits M. Cutting properties of common reed biomass. 8th International Scientific Conference: Engineering for Rural Development. Proceedings. May 28-29, 2009. Jelgava, 2009, pp. 207-211.

[3] Ancans D., Kakitis A., Nulle I. Parameters of stalk biomass cutter. 12th International Scientific Conference: Engineering for Rural Development. Proceedings. May 23-24, 2013. Jelgava, 2013, pp. 516-520.

[4] Kronbergs A., Kronbergs E., Rozinskis R. Size reduction of common reeds for biofuel production. 11th International Scientific Conference: Engineering for Rural Development. Proceedings. May 24-25, 20012. Jelgava, 2012, pp. 257-261. 
[5] Kronbergs A., Kronbergs E., Smits M., Siraks E. Investigation of common reed cutting methods. 4th International Conference TAE 2010: Trends in Agricultural Engineering 2010. Proceedings. September 7-10, 2010. Prague, 2010, pp. 338-342.

[6] Smits M., Kronbergs A., Kronbergs E. Herbaceos energy crop shredding size determination. 9th International Scientific Conference: Engineering for Rural Development. Proceedings. May 2728, 2010. Jelgava, 2010, pp. 275-279.

[7] Du, Y., Wang, J., Lin, Y., Liu, Z., Yu, H., Yi, H. Estimating the aboveground biomass of Phragmitesaustralis (common reed) based on multi-source data. 38th Annual IEEE International Geoscience and Remote Sensing Symposium, IGARSS 2018July 22-27, 2018. Valencia, 2018, pp. 9241-9244.

[8] Cubars E., Noviks G. Evaluation of reed resources in Latvia and analysis of its use for energy production. Available at: http://jeb.co.in/journal_issues/201204_apr12_supp/paper_13.pdf

[9] Cubars E., Noviks G. Evaluation of reed resources in Kurzemes region in Latvia. Procceeding of International conference "Renewable energy and energy efficiency", May 28-30, 2012, Jelgava, Latvia, pp. 19-23.

[10]Edited by Guangnan Chen. Advances in Agricultural Machinery and Technologies. CRC Press Taylor \& Francis Group, 2018. 472p.

[11] Smits M., Kronbergs E. Energy consumption for reed biomass size reduction. International Scientific Conference: Research for Rural Development. Proceedings. May 19-22, 2004. Jelgava, 2004, pp. 28-31.

[12] Smits M., Kronbergs E. Stalk material cutting energy. International Scientific Conference: Research for Rural Development. Proceedings. May 19-22, 2005. Jelgava, 2005, pp. 56-60. 